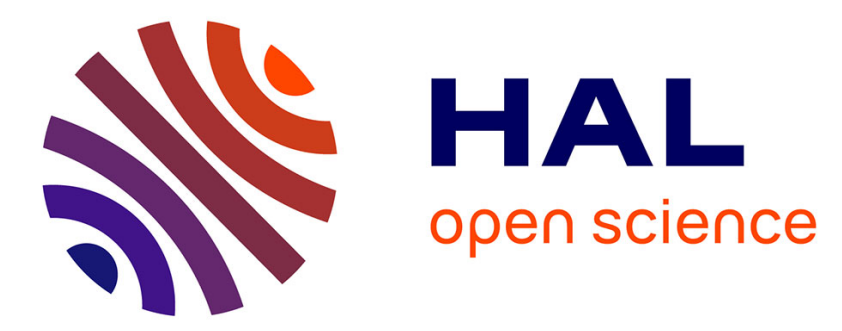

\title{
The alien's identity: consequences of taxonomic status for the international bumblebee trade regulations
}

Thomas Lecocq, Audrey Coppee, Denis Michez, Nicolas Brasero, Jean-Yves Rasplus, Irena Valterova, Pierre Rasmont

\section{- To cite this version:}

Thomas Lecocq, Audrey Coppee, Denis Michez, Nicolas Brasero, Jean-Yves Rasplus, et al.. The alien's identity: consequences of taxonomic status for the international bumblebee trade regulations. Biological Conservation, 2016, 195, pp.169-176. 10.1016/j.biocon.2016.01.004 . hal-01575596

\section{HAL Id: hal-01575596 \\ https://hal.univ-lorraine.fr/hal-01575596}

Submitted on 4 Dec 2020

HAL is a multi-disciplinary open access archive for the deposit and dissemination of scientific research documents, whether they are published or not. The documents may come from teaching and research institutions in France or abroad, or from public or private research centers.
L'archive ouverte pluridisciplinaire HAL, est destinée au dépôt et à la diffusion de documents scientifiques de niveau recherche, publiés ou non, émanant des établissements d'enseignement et de recherche français ou étrangers, des laboratoires publics ou privés.

\section{(ㅇ)(1) $\$$}

Distributed under a Creative Commons Attribution - NonCommercial - NoDerivatives| 4.0 


\section{The alien's identity: consequences of taxonomic status for the 2 international bumblebee trade regulations}

3 Thomas Lecocq ${ }^{\mathrm{a}, b^{*}}$, Audrey Coppée ${ }^{\mathrm{a}}$, Denis Michez ${ }^{\mathrm{a}}$, Nicolas Brasero ${ }^{\mathrm{a}}$, Jean-Yves Rasplus ${ }^{\mathrm{c}}$, Irena Valterovád, 4 and Pierre Rasmont ${ }^{\mathrm{a}}$

5 a: University of Mons, Research institute of Biosciences, Laboratory of Zoology, Place du Parc 20, 7000 Mons, Belgium

6 b: Research Unit Animal and Functionalities of Animal Products (URAFPA), University of Lorraine - INRA, 2 Avenue de la Forêt de Haye, BP 172, 54505, Vandoeuvre-lès-Nancy, France

c: INRA, UMR 1062 Centre de Biologie pour la Gestion des Populations, CS 30 016, F-34988 Montferrier / Lez cedex France

9 d: Academy of Sciences of the Czech Republic, Institute of Organic Chemistry and Biochemistry, Flamingovo nam 2, CZ-166 10 Prague,

10 Czech Republic

11 *Correspondence: Thomas Lecocq, Laboratory of Zoology, Research institute of Biosciences, University of Mons, B-7000, Mons, Belgium.

12 Tel: +3265373405. E-mail: thomas.lecocq@umons.ac.be

13 Short running title: The alien's identity

14 Highlights

15 The international trade fosters non-native taxon invasions

16 Several commonly traded species are taxon complexes

17 Preserving endemic taxa and differentiated populations require trade regulation

18 Such regulations need a resolved taxonomy and population structure

19 Integrative taxonomy provides guidelines for regulation of population importations

\section{Abstract}

21 The species international trade leads to multiple non-native invasions. Beside species invasions, commercial 22 exchanges may also contribute to translocation between closely related taxa or allopatric populations. 23 Consequently, preserving endemic taxa and specificity of local populations require to regulate commercial 24 translocations of species or populations. To be efficient such regulation needs a resolved taxonomy and a thorough analysis of the population structure of native taxa/populations. To provide guidelines for an efficient regulation of the trade of Bombus terrestris within its natural range, we analyzed its taxonomy and its population structure using an integrative taxonomic approach. Our results show that B. terrestris translocations involve two species, three subspecies, and several populations with weak differentiation. These different levels of differentiation imply specific and appropriate regulations of translocations with different levels of prioritization.

30 We ultimately assess the relevance of current policies and propose potentially efficient regulations for policymakers. Such integrative taxonomic approach should be used in other traded polytypic species.

\section{Keywords}

33 alien taxa; evolutionary significant units; integrative taxonomy; species international trade; bumblebee 
Invasions by non-native organisms is one of the main causes of the species endangerment throughout the world (Mack et al., 2000). These invasions are facilitated by the globalized trade of many species as food, pets, or biological control agents (Lowry et al., 2012; Perrings et al., 2010). While the invasions by non-native species have been the focus of abundant research (Courchamp et al., 2003; Mooney and Cleland, 2001; Simberloff et al., 2009), anthropogenic translocations between closely related taxa or allopatric populations have received much less attention (but see Rhymer and Simberloff, 1996). Yet, preserving endemic taxa and evolutionarily significant units (ESUs; i.e. allopatric or parapatric populations differentiated in neutral genetic markers and/or displaying locally adapted phenotypic traits) is a key step to preserve biodiversity and to ensure its long-term persistence (Conner and Hartl, 2004; Crowhurst et al., 2011; Frankham et al., 2010; Sgrò et al., 2011). The study of biological invasions resulting from the international trade of Bombus terrestris exemplifies this relatively low interest. Since the 1980s, B. terrestris hives have been massively produced to improve pollination of many crops (Velthuis and van Doorn, 2006). Each year, more than two million colonies are shipped throughout the world leading to deliberate releases or accidental escapes that foster B. terrestris establishments (Goulson, 2010; Inari et al., 2005; Murray et al., 2013; Velthuis and van Doorn, 2006). Most studies have focused on the invasions of B. terrestris out of its natural range (e.g. Inoue et al., 2008; Kanbe et al., 2008; Meeus et al., 2011) but not within (but see Ings et al., 2010; Murray et al., 2013). Yet, several B. terrestris subspecies (Fig. 1) have been reared and introduced in areas (Velthuis and van Doorn, 2006) where related populations or subspecies already occurred (e.g. B. terrestris audax in contact with B. terrestris dalmatinus in UK).

Preserving endemic taxa and specificity of differentiated populations requires a resolved taxonomy and population structure of native taxa/populations. This implies also to regulate commercial translocations and to forecast the potential consequences of translocations (i.e. the extent of invasion consequences partly depends on the phylogenetic relatedness between invasive and native organisms: non-native species introductions may lead to interspecific conflicts such as displacement of native species while invasions by non-native conspecific individuals could foster dilution of characters; Mooney and Cleland, 2001). However, determining (i) the most adapted species definition and, therefore, the most efficient species diagnostic traits or, even, (ii) the most efficient traits reflecting inter-population differentiation is challenging for most organisms (De Queiroz, 2007) including bumblebees (Lecocq et al., 2015d). Since bumblebees can display a large intraspecific or a low interspecific variability in morphology (Michener, 1990), alternative features (i.e. wing shape, DNA, or eco- 
63

chemical traits) have been used to discriminate entities leading however to controversial results (e.g. Aytekin et al., 2007; Brasero et al., 2015; Ellis et al., 2006). The recent integrative taxonomy based on unified species concept (De Queiroz, 2007) that considers multiple independent lines of evidence to evaluate taxonomic status (Schlick-Steiner et al., 2010) provides a solution to overcome such taxonomic issues as well as to identify interpopulation differentiation (Lecocq et al., 2015b, 2015d). This approach was applied to a partial sampling of $B$. terrestris populations suggesting that $B$. terrestris is a species complex with several differentiated subspecies (Lecocq et al., 2015b). These results question the relevance of current regulations of B. terrestris trade that mainly disregard the intra-B. terrestris differentiation or aim to protect populations (e.g. B. terrestris audax in UK) differentiated in character that potentially poorly reflect differentiation processes (i.e. hair body color pattern; Bertsch and Schweer, 2012).

In this paper, we aim to provide guidelines for efficient regulations of B. terrestris translocation within its natural range according to a resolved taxonomy and population structure within B. terrestris.

\section{Materials and methods}

\subsection{Studied species and sampling}

Bombus terrestris has been considered by some taxonomists as a species complex while others recognized only one species (Rasmont et al., 2008). Beside, the first studies based on a unsuitable diagnostic character (i.e. color pattern; Carolan et al., 2012), barcoding studies suggested that all B. terrestris taxa are conspecific (Bertsch, 2010; Williams et al., 2012b). In contrast, studies of the cephalic labial gland secretions (CLGS), a reproductive trait involved in the bumblebee pre-mating recognition reported significant differentiations between some subspecies that question their conspecificity (Coppée et al., 2008; Lecocq et al., 2015c, 2013). Recently, an integrative approach based on differentiation in multiple genetic markers and CLGS composition suggested that B. terrestris included two species (B. terrestris and B. xanthopus) but only six taxa of the complex were analyzed (Lecocq et al., 2015b).

Here, we extended the integrative taxonomic studies of Lecocq et al. (2015b) to the nine B. terrestris taxa (recognized as subspecies by Rasmont et al., 2008 according to their diagnostic hair body color pattern). Between 2004 and 2013, we sampled (i) 147 specimens of B. terrestris africanus from Morocco, B. terrestris audax from England and Ireland, B. terrestris calabricus from Sicily, B. terrestris canariensis from Canary Islands, B. terrestris dalmatinus from SE France, Aegean islands and Turkey, B. terrestris lusitanicus from SW 
Europe and Madeira, B. terrestris sassaricus from Sardinia, B. terrestris terrestris from $\mathrm{W}$ and $\mathrm{N}$ Europe, and B. terrestris xanthopus from Corsica, and (ii) six specimens of B. terrestris dalmatinus from two colonies produced by Biobest bvba (Westerlo, Belgium) in 2007 and 2008 (Appendix Table A1). We considered all taxa without $a$ priori taxonomic status and referred to them as africanus, audax, calabricus, canariensis, dalmatinus, lusitanicus, sassaricus, terrestris, and xanthopus. Among these taxa, five are or were used in the international trade: canariensis, dalmatinus, sassaricus, terrestris, and xanthopus. Taxa with a large-range distribution were sampled in a higher number of locations (Appendix Table A1). Moreover, we tried to sample at different locations (distant by more than $10 \mathrm{~km}$ corresponding to the maximum male and queen flight distances; Kraus et al., 2009; Lepais et al., 2010) to avoid sampling family members but we failed for africanus, calabricus, and canariensis (for the last taxa, its restricted geographic range does not allow sampling at distant places). We considered the phylogenetically related species $B$. ignitus as outgroup. We killed specimens by freezing them at $20^{\circ} \mathrm{C}$.

\subsection{COI sequence-based species delimitation for the DNA taxonomic analyses}

104 We sequenced the mitochondrial cytochrome oxidase 1 (COI) gene commonly used to study the interspecific relationship in bumblebees (Williams et al., 2012b). We followed the total DNA extraction protocol, polymerase chain reaction amplification reactions, sequencing procedures, and alignment method described in Lecocq et al. (2015b). Sequences were deposited in GenBank (Appendix Table A1). The final molecular dataset spanned $849 \mathrm{bp}$.

We delimited species based on the COI sequences using the Bayesian implementation of the general mixed Yule-coalescent (bGMYC) method integrating the uncertainty related to phylogenetic inference (Reid and Carstens, 2012), recently underlined as an efficient species delimitation method compared to alternative single molecular- based methods (i.e. barcode, GMYC; see Dellicour and Flot, 2015; Lecocq et al., 2015d). For each pair of DNA sequences, this method estimates the posterior probability that specimens are conspecific. The

114 probability that a lineage was conspecific with other lineages was here estimated by reporting ranges of posterior 115 probabilities among sequences from different lineages. The bGMYC method relies on the prediction that 116 independent evolution leads to the appearance of distinct genetic clusters (i.e. monophyly), separated by longer internal branches. A range of probabilities $>0.90$ was considered as strong evidence that the groups compared

118 were conspecific while a range of probabilities $<0.05$ strongly suggested that the groups compared were not conspecific (Reid and Carstens, 2012). Intermediate probabilities were interpreted as indicating non-significance; 
in these cases the method was not able to confirm if the groups compared were conspecific or were not conspecific (Reid and Carstens, 2012). Since the bGMYC algorithm requires several phylogenetic ultrametric trees, we used BEAST 1.7.4 (using a GTR+I+G; number of gamma categories = 4; partition into codon base positions, unlink substitution rate parameters across codon positions and unlink base frequencies across codon

124 positions; a random starting tree; four chains with mixed-models; under Yule process model) (Drummond et al., 125 2012) with a phylogenetic clock model to generate a posterior distribution of trees (length of the MCMC chain: 1 126 billion generations). We used Tracer version 1.5 (Rambaut and Drummond, 2007) to assess convergence between the chains. The bGMYC analysis was based on 1000 trees sampled every 10000 generations from the BEAST analysis. For each of these 1000 trees, the MCMC was made of 100000 generations, discarding the first 90000 as burn-in and sampling every 100 generations.

\subsection{Male chemical reproductive traits for chemo-taxonomical analyses}

131 We analyzed the CLGS commonly used as chemical markers for resolving species status (Lecocq et al., 2015d).

132 CLGS are a complex mixture of compounds, with several main components (i.e. compounds that have the 133 highest relative proportion among all compounds of CLGS at least in one individual of the taxa) (Lecocq et al., 134 2015d). We analyzed CLGS (extracted by hexane) by (i) gas chromatography-flame ionization detector (GC/FID 135 for quantification), (ii) gas chromatography-mass spectrometry (GC/MS for qualification) and (iii) dimethyl 136 disulfide derivatization (DMDS for the double bound position determination) following protocol and GC/FID, 137 GC/MS, and DMDS conditions described in Lecocq et al. (2015b). We used quantification procedure, relative 138 amount calculation, and alignment method described in Lecocq et al. (2015b).The final chemical dataset spanned 13968 compounds (Appendix Table A2).

140 We performed statistical analyses in R (R Development Core Team, 2013) to detect CLGS differentiations 141 among taxa. We transformed data $(\log (x+1))$ to reduce the great difference of abundance between compounds in 142 highly and low concentration. We compared the CLGS composition between individuals with a clustering 143 analysis performed with UPGMA (unweighted pair group method with arithmetic mean) clustering method on 144 Pearson r Correlation distances matrix (R-package ape, Paradis et al., 2004). We assessed the uncertainty in 145 hierarchical cluster analysis with p-values calculated via multiscale bootstrap resampling with 100000 number 146 of bootstrap sample size (R-package pvclust, Suzuki and Shimodaira 2011). We assessed CLGS differentiations 147 between groups detected in cluster by performing multiple response permutation procedure (MRPP) (R-package 148 vegan, Oksanen et al., 2011). The MRPP is a nonparametric, multivariate procedure that tests the null hypothesis 
149 of no difference between groups. MRPP has the advantage of not requiring distributional assumptions (such as

150 multivariate normality and homogeneity of variances). To determine compounds specific and regular to each

151 group defined by the clustering analyses and the MRPP (indicator compounds), we used the indicator value

152 (IndVal) method (Dufrêne and Legendre, 1997). We evaluated the statistical significance of a compound as an

153 indicator at the 0.01 level with a randomization procedure.

154 Reproductive trait differentiation can result in low regional variation with minor behavioral consequences (e.g.

155 Vereecken, Mant, \& Schiestl, 2007) or in the rise of reproductive isolation barrier (e.g. Martens, 1996). The

156 CLGS differentiation consequences remain poorly predictable without field observations or ethological tests

157 (most of the time unavailable; Lecocq et al., 2013). Nevertheless, the CLGS comparison between closely related

158 bumblebee taxa with a recognized species status support that the interspecific differentiation involves changes in

159 main compounds (e.g. Bertsch \& Schweer 2012; Calam 1969). Therefore, we considered a significant (assessed

160 by MRPP) CLGS differentiation along with a main compound divergence (assessed by IndVal) as a strong

161 indicator of potential ethological consequences for pre-mating recognition.

\subsection{Data integration and decision framework of taxonomic status}

163 We used the decision framework developed by Lecocq et al. (2015b,d). We considered that a taxon deserved a 164 species status with a high degree of certainty if the taxon (i) was genetically differentiated (COI original 165 haplotypes), (ii) was not significantly conspecificity with other taxa in bGMYC analyses (probability < 0.9 to be 166 conspecific), (iii) was significantly differentiated in CLGS compositions, and (iv) was differentiated in the main 167 CLGS compounds (see argumentation in Lecocq et al., 2015b). We assigned subspecies status if there were 168 divergences in some but not all operational criteria according to the subspecies definition proposed by 169 Hawlitschek et al. (2012). We considered color pattern specificity as a further evidence of differentiation when 170 there is differentiation in at least one operational criterion but we did not consider divergence observed only 171 color pattern as reflecting a differentiation process because of limitations of this trait as diagnostic criterion 172 (Bertsch and Schweer, 2012a; Carolan et al., 2012).

\section{3.Results}

174 3.1.COI sequence-based species delimitation for the DNA taxonomic analyses 
175 COI phylogenetic analysis detected five monophyletic groups that were strongly supported (Fig. 2A; see 176 sequence divergences between taxa in Appendix Table A3 ): (i) B. ignitus, (ii) xanthopus, (iii) one clade 177 including audax, calabricus, several dalmatinus, few lusitanicus, and most of terrestris specimens, (iv) one 178 clade with africanus and canariensis (in two different lineages), (v) one clade with other dalmatinus from 179 bumblebee breeders, most of lusitanicus, sassaricus (in one distinct clade), and other dalmatinus, terrestris (Fig. 180 2A). Xanthopus was resolved as the sister taxa of all B. terrestris taxa (Fig. 2A). The bGMYC analysis showed 181 probabilities of conspecificity of 0 between outgroup and ingroup (Appendix Table A4). Within the ingroup, the 182 bGMYC analysis showed two entities with low probabilities $(<0.05)$ to be conspecific: xanthopus versus all 183 other B. terrestris taxa (Appendix Table A4). At least the bGMYC analysis revealed that all other B. terrestris 184 taxa have a non-significant probability (from 0.9 to 0.05 but most of probabilities were near the hetero185 specificity threshold; i.e. 0.06) or a high probability (>0.95) to be conspecific (Appendix Table A4).

3.2.Male chemical reproductive traits for chemo-taxonomical analyses

187 The cluster analysis of our CLGS data matrix (B. ignitus and all B. terrestris subspecies) revealed four strongly 188 supported (bootstrap > 95) groups (Fig. 2B): G1 (xanthopus), G2 (B. ignitus), G3 (including three subgroups: two groups of dalmatinus and one group of sassaricus), G4 (all other dalmatinus and all other B. terrestris taxa)

190 that included two distinct sub-groups (all africanus and canariensis specimens respectively formed two 191 differentiated sub-groups). Five specimens did not cluster with other specimens (ungrouped specimens). The 192 examination of the wing condition of the ungrouped individuals indicated that these specimens were obviously old specimens (Lecocq et al., 2011) that are known to show different CLGS composition and to be lesser 194 attractive to females (Coppée et al., 2011; Žáček et al., 2009).

195 Our global MRPP confirmed these intergroup differentiations ( $\mathrm{T}$ [the overall weighted mean of group mean 196 distances] $=0.33$, A [chance-corrected estimate of the proportion of the distances explained by group 197 identity $]=0.28$, $p$-value $<0.01)$. Pairwise MRPP supported divergences between these groups $(\mathrm{G} 1$ versus $\mathrm{G} 2$ : T= $1980.17, \mathrm{~A}=0.58$, p-value $<0.01 ; \mathrm{G} 1$ versus $\mathrm{G} 3: \mathrm{T}=0.26, \mathrm{~A}=0.35$, p-value $<0.01$; $\mathrm{G} 1$ versus $\mathrm{G} 4: \mathrm{T}=0.36, \mathrm{~A}=0.11, p$ 199 value $<0.01 ; \mathrm{G} 2$ versus $\mathrm{G} 3: \mathrm{T}=0.21, \mathrm{~A}=0.41$, -value $<0.01 ; \mathrm{G} 2$ versus $\mathrm{G} 4: \mathrm{T}=0.35, \mathrm{~A}=0.16, p$-value $<0.01 ; \mathrm{G} 3$ 200 versus $\mathrm{G} 4: \mathrm{T}=0.35, \mathrm{~A}=0.16$, -value $<0.01)$. We also checked that the two distinct groups included in $\mathrm{G} 4$ that correspond to africanus and canariensis with Pairwise MRPP (africanus versus G4 expected africanus and canariensis: $\mathrm{T}=0.35, \mathrm{~A}=0.03$, p-value $<0.01$; canariensis versus $\mathrm{G} 4$ expected africanus and canariensis: $\mathrm{T}=$ 
204 (G1, G2, G3, and G4 divided between africanus, canariensis, and other taxa), the main compounds are (i) G1: 205 dihydrofarnesol, hexadecenyl hexadecenoate, icos-17-enal, tricosane, (ii) G2: dihydrofarnesol and octadec-13206 en-1-ol, (iii) G3: dihydrofarnesol, (iv) G4 without canariensis and africanus: dihydrofarnesol, dihydrofarnesyl dodecanoate and ethyl dodecanoate, (v) africanus: dihydrofarnesol, dihydrofarnesyl dodecanoate, ethyl 208 dodecanoate, and geranylcitronellol, (vi) canariensis: dihydrofarnesol. For each group, the IndVal method revealed several significantly and strongly indicator compounds (IndVal value >0.90) including main compounds only in xanthopus and B. ignitus (Appendix Table A2).

\subsection{Taxonomic status}

212 Species status is assigned to B. ignitus (outgroup) and to B. xanthopus according to their genetic differentiation, 213 their non-significant conspecificity with other taxa in bGMYC analyses (probability $<0.9$ to be conspecific) and 214 their specific CLGS composition (including main compounds) (Table 1). All other taxa are included in one 215 species (Table 1). Africanus, canariensis, and sassaricus were differentiated in several operational criteria 216 (specific CLGS composition, without main compound changes, and private haplotypes). Consequently, the 217 subspecies status is confirmed for these taxa. In contrast, audax, calabricus, dalmatinus (wild and commercial), 218 lusitanicus, and terrestris were differentiated only in color pattern (Table 1).

\section{4.Discussion}

\subsection{Solving the taxonomic status and the population structure}

221 The integrative taxonomy approach applied previously to a subset of $B$. terrestris taxa (Lecocq et al., 2015b) and extended here to all $B$. terrestris entities strongly suggests the existence of two species: $B$. xanthopus and $B$. terrestris. Consequently, the species status of B. xanthopus proposed by Lecocq et al. (2015b) is confirmed according to COI and CLGS differentiations (Tab. 1). While previous studies based on single criterion (Bertsch and Schweer, 2012a; Williams et al., 2012b) do not regards these divergences as deserving a species status (Lecocq et al., 2015b), we assume that the concordance of differentiation in multiple diagnostic traits reflects a speciation process. The conspecificity of all other B. terrestris entities (including new taxa compared to Lecocq et al., 2015b) assessed through our our multi-criterion approach agrees with all recent single criterion-based taxonomic studies (Bertsch and Schweer, 2012a; Rasmont et al., 2008; Williams et al., 2012b). 
At the intraspecific level, a geographic differentiation process is detected for some allopatric B. terrestris taxa

231 (Fig. 2, Tab. 1). As observed in several organisms such as amphibians (Bisconti et al., 2011), insects (Damgaard,

232 2005), or mammals (Evin et al., 2011), the South West islands and North Africa host highly differentiated 233 populations. These populations are genetically (in the studied mitochondrial DNA marker) (Fig. 2; previously 234 observed by Estoup et al., 1996) and ecologically (Rasmont et al., 2008) differentiated and deserve a subspecies status (sensu Hawlitschek et al., 2012): B. terrestris africanus, B. terrestris canariensis, and B. terrestris sassaricus. In contrast, other insular populations (audax and Madeira lusitanicus) do not display such level of differentiations (Fig. 2; also previously observed by Widmer et al., 1998). A persistent gene flow between European mainland and British Islands as suggested by bumblebee channel crossings (Goulson et al., 2011; Mikkola, 1978) could explain the observed pattern for audax. Further studies on a putative gene flow between UK and Europe are needed to assess this hypothesis. For the Madeira lusitanicus, the lack of phenotypic and genetic differentiation with continental lusitanicus contradicts the strong geographic isolation of the island and suggests an ancient human-related colonization of the island from the Iberian Peninsula (as far as we know there are no bumblebee traded for pollination in Madeira has not been imported for commercial purpose in Madeira).

244 Considering continental taxa, calabricus, wild dalmatinus, lusitanicus, and terrestris do not display 245 differentiation in COI or CLG (similar results in Bertsch and Schweer, 2012; Estoup et al., 1996). This may 246 reflect that (i) a priori classification based on color patterns poorly reflects the genetic differentiation (Lecocq et 247 al., 2015a), (ii) there is no geographic structure across the mainland (Bertsch and Schweer, 2012a). Although 248 classification based on color patterns has been critized (Bertsch and Schweer, 2012a; Lecocq et al., 2015a), the 249 second hypothesis appears more likely since there is no alternative coherent population structure in our analyses 250 (Fig. 2) regardless of the a priori taxonomic classification. Indeed, our analyses detects (i) two types of CLGS 251 within wild sympatric dalmatinus populations (Fig.2b) and (ii) two genetically distinct and broadly sympatric groups each with wild dalmatinus, lusitanicus, and terrestris specimens (i.e. the two CLGS groups observed in dalmatinus do not match with the two genetic groups observed in this taxon) but these groups do not correspond to a geographic structure. Moreover, since these distinct groups are not related to other phenotypic or ecologic differentiations, this observation is most likely a consequence of a smaller sampling to evaluate the whole genetic/CLGS variability of these widespread taxa (Lecocq et al., 2015d) and/or, for the CLGS, a result from an age differentiation that impacts the chemical composition (Žáček et al., 2009). This hypothesis is further supported by several inter-taxa geographic clines observed on mainland (Bertsch and Schweer, 2012a; Rasmont et al., 2008). Alternatively, the lack of differentiation between most of B. terrestris entities (including audax) 
could result from the genetic homogenization linked to the importation of commercial B. terrestris during the 261 last 20 years and that may have erased the past population structure (Velthuis and van Doorn, 2006). Indeed, all 262 samples analyzed (and those used in previous works; e.g. Bertsch and Schweer, 2012; Estoup et al., 1996) were sampled long after the start of the commercialization of B. terrestris. Pre-commercialization samples (i.e. 264 museum specimens) need to be analyzed to test this hypothesis. At least, we cannot exclude that diagnostic characters chosen could be relatively invariant/inconsistently differentiated traits within B. terrestris (could be likely for CLGS; i.e. Bertsch and Schweer, 2012a; Lecocq et al., 2015a; but the COI marker has been successfully used to solve the population structure of many bumblebee species, e.g. Dellicour et al., 2015; Duennes et al., 2012). This could hide some local adaptations and differentiation process since the accuracy of the integrative approach is depending on selected characters (Lecocq et al., 2015d). This may likely happen for dalmatinus and lusitanicus that display ecological, ethological, and phenological specificity (e.g. larger colony size, shorter setae, aestivation; Peat et al., 2005; Rasmont et al., 2008; Velthuis and van Doorn, 2006) suggesting a differentiation process while other undifferentiated taxa are similar for these features. Taking into account these specificity and the fact that dalmatinus is the most used taxa for international trade, we consider them as an

274 ESU within $B$. terrestris. These taxa and other poorly differentiated taxa should be analyzed through alternative 275 traits and further analyses (e.g. microsatellite analyses, Duennes et al., 2012) to detected potential population 276 structure.

277 Considering commercial dalmatinus strain, the lack of differences in studied characters suggest that the breeding 278 process did not lead to differentiation process but further potential differentiation of traded strain should be 279 investigated.

281 Given the important advantages of traded bumblebee for crop pollinations (Velthuis and van Doorn, 2006), a 282 complete ban of international B. terrestris trade is not feasible. Therefore, efficient strategies to manage the trade 283 of B. terrestris are needed to reconcile biological conservation and economic reality. This implies mainly 284 regulations of translocations/importations of B. terrestris taxa (Velthuis and van Doorn, 2006).

285 Nowadays, such regulation mostly concerns importation of B. terrestris out of its natural range (e.g. Australia; 286 North America; Velthuis and van Doorn, 2006) in order to avoid interspecific competitions and the highly 287 problematic pathogen spillover due to importations of exotic pollinators (Murray et al., 2013; Schmid-Hempel et 
al., 2014). Given the new species status of $B$. xanthopus and to protect this endemic species, importations of $B$. terrestris should be prohibited in Corsica. Moreover the high percentage of endemism in the Corsican fauna of bumblebees (several endemic species and subspecies; Lecocq et al., 2015b) reinforces the necessity of such a prohibition.

Within the natural distribution of $B$. terrestris, measures to regulate its translocation have been taken in Canary Islands, Israel, Norway, Turkey, and UK only (Velthuis and van Doorn, 2006). For the here assessed B. terrestris subspecies (B. terrestris africanus, B. terrestris canariensis, and B. terrestris sassaricus), regulations promoted by Canary Islands authorities provide an example of adapted regulations that should be extended to Sardinia and North Africa. These regulations only allow using the local subspecies (B. terrestris canariensis) for crop pollination (Velthuis and van Doorn, 2006). However, current legislation within Canary Islands allow the breeding of the local subspecies out of its natural range and the subsequent importation of colonies (Velthuis and van Doorn, 2006) that could lead to pathogen spillovers (Murray et al., 2013). Prohibition of all bumblebee importations to isolated lands could hamper such potential issues. Consequently, local crop pollination would require productions of local populations (Williams et al., 2012a) but such productions can only become economically viable if the size of the local market for pollination is substantial (e.g. see the decision of bumblebee breeders to leave the Norwegian market after importation prohibition; Velthuis and van Doorn, 2006). Since Canary Islands, North Africa, and Sardinia export a huge amount of crops commercially pollinated by bumblebees (Velthuis and van Doorn, 2006), complete interdiction of bumblebee importation should foster development of local production leading to beneficial effect for biological conservation and local economy.

Considering other B. terrestris populations, one could argue that the importation of allopatric bumblebee populations within British Islands, Europe, and West Asia is not an issue according to the lack of differentiations in our integrative taxonomic approach. However, since this lack of differentiation could be a consequence of an undergoing genetic homogenization of $B$. terrestris or could be linked to the limitation of our integrative approach (see before), a precautionary approach should be promoted pending further analyses on the $B$ terrestris intraspecific structure. While the Israeli, Norwegian, Turkish, UK laws prohibiting importation and use of nonnative strains remain the safest approach, its economic viability definitively depends on the size of local market 314 for pollination (Velthuis and van Doorn, 2006). Minimum regulation should concern dalmatinus and lusitanicus 315 ESU since they (i) display the most conspicuous specificity within "undifferentiated taxa" and (ii) are the most used strains in international trade. Their importation should be restricted to its natural range (Rasmont et al., 

also the few evidence of differentiations previously recorded and the observed clines in natura (Bertsch and

319 Schweer, 2012a; Estoup et al., 1996; Rasmont et al., 2008; Widmer et al., 1998; Williams et al., 2012b), importations between audax, calabricus, and terrestris ranges could be alternatively prohibited or authorized but not encouraged taking into account the risks of pathogen spillover.

Beside this first recommendation for regulation of $B$. terrestris trade, a major uncertainty remains in the identity of the commercial strains. Our analyses show the lack of specificity for a commercial strain compared to wild dalmatinus but similar analyses should be conduct on other commercial strains. Indeed, commercial imports can involve individuals (i) bred for several generations in non-natural conditions to generate strains with specific ecological requirements (different from natural populations) or (ii) resulting from intersubspecific/interpopulation hybridization during the breeding process depending on the breeding company (Velthuis and van Doorn, 2006). In this case, commercial strains would be considered as aliens everywhere.

\subsection{Further applications}

330 Here applied to the $B$. terrestris trade, the definition of guidelines for the regulation of species trade through integrative taxonomy assessments should be extended to other traded organisms exhibiting intraspecific polymorphism (e.g. Australian parrots or rice; Song \& Carter 1996; Low 2014). Such assessment of taxonomic and population structure (including ESUs highlighting) can provide pragmatic guidelines to legislate for smart regulation that reconciles biological conservation and economic reality. Nevertheless, the efficiency of such regulations requires that the taxonomic identity or the geographic origin of traded strains should be assessed and

336 provided by trade companies.

\section{Acknowledgements}

338 We thank R. De Jonghe, N. E. Raine, L. Chittka, and Biobest bvba for providing the biological material. TL was post-doc funded by the Belgian Scientific Politic (BELSPO, BR/132/A1/BELBEES). Financial support was

340 provided by the European Community's Seventh Framework Programme (FP7/2007-2013) under grant agreement no244090, STEP Project (Status and Trends of European Pollinators, www.step-project.net) and the

342 Czech Science Foundation (grant No. 14-04291S). This project was supported by the network Bibliothèque du 343 Vivant funded by the CNRS, the Muséum National d'Histoire Naturelle and the Institut National de la Recherche 344 Agronomique. 
346 Supplementary data associated with this article can be found in the online version.

347

348

350

\section{References}

Aytekin, A.M., Terzo, M., Rasmont, P., Cagatay, N., 2007. Landmark based geometric morphometric analysis of wing shape in Sibiricobombus Vogt (Hymenoptera : Apidae : Bombus Latreille). Ann. Soc. Entomol. Fr. $43,95-102$.

Bertsch, A., 2010. A phylogenetic framework for the bumblebee species of the subgenus Bombus sensu stricto based on mitochondrial DNA markers, with a short description of the neglected taxon B. minshanicola Bischoff, 1936 n. status. Beitr. Ent. 60, 471-487.

Bertsch, A., Schweer, H., 2012. Cephalic labial gland secretions of males as species recognition signals in bumblebees: are there really geographical variations in the secretions of the Bombus terrestris subspecies? Beitr. Ent. 62, 103-124.

Bisconti, R., Canestrelli, D., Nascetti, G., 2011. Genetic diversity and evolutionary history of the Tyrrhenian treefrog Hyla sarda (Anura: Hylidae): Adding pieces to the puzzle of Corsica-Sardinia biota. Biol. J. Linn. Soc. $103,159-167$.

Brasero, N., Martinet, B., Urbanová, K., Valterová, I., Torres, A., Hoffmann, W., Rasmont, P., Lecocq, T., 2015. First chemical analysis and characterization of the male species-specific cephalic labial-gland secretions of south american bumblebees. Chem. Biodivers. 12, 1535-1546.

Calam, D.H., 1969. Species and Sex-specific Compounds from the Heads of Male Bumblebees (Bombus spp.). Nature 221, 856-857.

Carolan, J.C., Murray, T.E., Fitzpatrick, Ú., Crossley, J., Schmidt, H., Cederberg, B., McNally, L., Paxton, R.J., Williams, P.H., Brown, M.J.F., 2012. Colour patterns do not diagnose species: quantitative evaluation of a DNA barcoded cryptic bumblebee complex. PLoS One 7, e29251.

Conner, J.K., Hartl, D.L., 2004. A Primer of Ecological Genetics. Sinauer Associates, Sunderland, MA.

Coppée, A., Mathy, T., Cammaerts, M.-C., Verheggen, F.J., Terzo, M., Iserbyt, S., Valterová, I., Rasmont, P., 2011. Age-dependent attractivity of males' sexual pheromones in Bombus terrestris (L.) [Hymenoptera, 
Apidae]. Chemoecology 21, 75-82.

Coppée, A., Terzo, M., Valterová, I., Rasmont, P., 2008. Intraspecific variation of the cephalic labial gland secretions in Bombus terrestris (L.) (Hymenoptera: Apidae). Chem. Biodivers. 5, 2654-2661.

Courchamp, F., Chapuis, J.-L., Pascal, M., 2003. Mammal invaders on islands: impact, control and control impact. Biol. Rev. 78, 347-383.

Crowhurst, R.S., Faries, K.M., Collantes, J., Briggler, J.T., Koppelman, J.B., Eggert, L.S., 2011. Genetic relationships of hellbenders in the Ozark highlands of Missouri and conservation implications for the Ozark subspecies (Cryptobranchus alleganiensis bishopi). Conserv. Genet. 12, 637-646.

Damgaard, J., 2005. Genetic diversity, taxonomy, and phylogeography of the western Palaearctic water strider Aquarius najas (DeGeer) (Heteroptera: Gerridae). Insect Syst. Evol. 36, 395-406.

De Queiroz, K., 2007. Species concepts and species delimitation. Syst. Biol. 56, 879-886.

Dellicour, S., Flot, J.-F., 2015. Delimiting Species-Poor Data Sets using Single Molecular Markers: A Study of Barcode Gaps, Haplowebs and GMYC. Syst. Biol. 64, 900-908.

Dellicour, S., Michez, D., Mardulyn, P., 2015. Comparative phylogeography of five bumblebees: impact of range fragmentation, range size and diet specialization. Biol. J. Linn. Soc. 116, 926-939.

Drummond, A.J., Suchard, M.A., Xie, D., Rambaut, A., 2012. Bayesian phylogenetics with BEAUti and the BEAST 1.7. Mol. Biol. Evol. 29, 1969-1973.

Duennes, M.A., Lozier, J.D., Hines, H.M., Cameron, S.A., 2012. Geographical patterns of genetic divergence in the widespread Mesoamerican bumble bee Bombus ephippiatus (Hymenoptera: Apidae). Mol. Phylogenet. Evol. 64, 219-31.

Dufrêne, M., Legendre, P., 1997. Species assemblages and indicator species: The need for a flexible asymmetrical approach. Ecol. Monogr. 67, 345-366.

Ellis, J.S., Knight, M.E., Carvell, C., Goulson, D., 2006. Cryptic species identification: a simple diagnostic tool for discriminating between two problematic bumblebee species. Mol. Ecol. Notes 6, 540-542.

Estoup, A., Solignac, M., Cornuet, J.-M., Goudet, J., Scholl, A., 1996. Genetic differentiation of continental and island populations of Bombus terrestris (Hymenoptera: Apidae) in Europe. Mol. Ecol. 5, 19-31. 
Evin, A., Nicolas, V., Beuneux, G., Toffoli, R., Cruaud, C., Couloux, A., Pons, J.-M., 2011. Geographical origin and endemism of Corsican Kuhl's pipistrelles assessed from mitochondrial DNA. J. Zool. 284, 31-39.

Facon, B., Crespin, L., Loiseau, A., Lombaert, E., Magro, A., Estoup, A., 2011. Can things get worse when an invasive species hybridizes? The harlequin ladybird Harmonia axyridis in France as a case study. Evol. Appl. 4, 71-88.

Frankham, R., Ballou, J.D., Briscoe, D.A., 2010. Introduction to Conservation Genetics, 2nd ed. Cambridge University Press, Cambridge, UK.

Goulson, D., 2010. Impacts of non-native bumblebees in Western Europe and North America. Appl. Entomol. Zool. 45, 7-12.

Goulson, D., Kaden, J.C., Lepais, O., Lye, G.C., Darvill, B., 2011. Population structure, dispersal and colonization history of the garden bumblebee Bombus hortorum in the Western Isles of Scotland. Conserv. Genet. $12,867-879$.

Hawlitschek, O., Nagy, Z.T., Glaw, F., 2012. Island evolution and systematic revision of Comoran snakes: why and when subspecies still make sense. PLoS One 7, e42970.

Inari, N., Nagamitsu, T., Kenta, T., Goka, K., Hiura, T., 2005. Spatial and temporal pattern of introduced Bombus terrestris abundance in Hokkaido, Japan, and its potential impact on native bumblebees. Popul. Ecol. 47, 77-82.

Ings, T.C., Ings, N.L., Chittka, L., Rasmont, P., 2010. A failed invasion? Commercially introduced pollinators in Southern France. Apidologie 41, 1-13.

Inoue, M.N., Yokoyama, J., Washitani, I., 2008. Displacement of Japanese native bumblebees by the recently introduced Bombus terrestris (L.) (Hymenoptera: Apidae). J. Insect Conserv. 12, 135-146.

Kanbe, Y., Okada, I., Yoneda, M., Goka, K., Tsuchida, K., 2008. Interspecific mating of the introduced bumblebee Bombus terrestris and the native Japanese bumblebee Bombus hypocrita sapporoensis results in inviable hybrids. Naturwissenschaften 95, 1003-1008.

Kraus, F.B., Wolf, S., Moritz, R.F.A., 2009. Male flight distance and population substructure in the bumblebee Bombus terrestris. J. Anim. Ecol. 78, 247-252. 
interspecific and intraspecific integrative taxonomic assessment of the widespread pollinator Bombus pascuorum Scopoli 1763 (Hymenoptera: Apidae). Syst. Entomol. 40, 881-888.

Lecocq, T., Brasero, N., Meulemeester, T. De, Michez, D., Dellicour, S., Lhomme, P., Jonghe, R. De, Valterová, I., Urbanová, K., Rasmont, P., 2015b. An integrative taxonomic approach to assess the status of Corsican bumblebees : implications for conservation. Anim. Conserv. 18, 236-248.

Lecocq, T., Coppée, A., Mathy, T., Lhomme, P., Cammaerts-Tricot, M.-C., Urbanová, K., Valterová, I., Rasmont, P., 2015c. Subspecific differentiation in male reproductive traits and virgin queen preferences, in Bombus terrestris. Apidologie 46, 595-605.

Lecocq, T., Dellicour, S., Michez, D., Dehon, M., Dewulf, A., De Meulemeester, T., Brasero, N., Valterová, I., Rasplus, J.-Y., Rasmont, P., 2015d. Methods for species delimitation in bumblebees (Hymenoptera, Apidae, Bombus): towards an integrative approach. Zool. Scr. 44, 281-297.

Lecocq, T., Lhomme, P., Michez, D., Dellicour, S., Valterová, I., Rasmont, P., 2011. Molecular and chemical characters to evaluate species status of two cuckoo bumblebees: Bombus barbutellus and Bombus maxillosus (Hymenoptera, Apidae, Bombini). Syst. Entomol. 36, 453-469.

Lecocq, T., Vereecken, N.J., Michez, D., Dellicour, S., Lhomme, P., Valterová, I., Rasplus, J.-Y., Rasmont, P., 2013. Patterns of genetic and reproductive traits differentiation in mainland vs. Corsican populations of bumblebees. PLoS One 8, e65642.

Lepais, O., Darvill, B., O’Connor, S., Osborne, J.L., Sanderson, R.A., Cussans, J., Goffe, L., Goulson, D., 2010. Estimation of bumblebee queen dispersal distances using sibship reconstruction method. Mol. Ecol. 19, 819-831.

Low, B.W., 2014. The global trade in native Australian parrots through Singapore between 2005 and 2011: a summary of trends and dynamics. EMU 114, 277-282.

Lowry, E., Rollinson, E.J., Laybourn, A.J., Scott, T.E., Aiello-Lammens, M.E., Gray, S.M., Mickley, J., Gurevitch, J., 2012. Biological invasions: a field synopsis, systematic review, and database of the literature. Ecol. Evol. 3, 182-96.

Mack, R.N., Simberloff, D., Lonsdale, W.M., Evans, H., Clout, M., Bazzaz, F.A., 2000. Biotic invasions: Causes, epidemiology, global consequences, and control. Ecol. Appl. 10, 689-710. 
Martens, J., 1996. Vocalizations and speciation of Palearctic birds, in: Kroodsma, D.E., Miller, E.H. (Eds.), Ecology and Evolution of Acoustic Communication in Birds. Comstock Publishing, Ithaca, New York, pp. $221-240$.

Meeus, I., Brown, M.J.F., De Graaf, D.C., Smagghe, G., 2011. Effects of Invasive Parasites on Bumble Bee Declines. Conserv. Biol. 25, 662-671.

Michener, C.D., 1990. Classification of the Apidae (Hymenoptera). Univ. Kansas Nat. Hist. Museum Spec. Publ. $54,75-164$.

Mikkola, K., 1978. Spring migrations of wasps and bumble bees on the southern coast of Finland (Hymenoptera, Vespidae and Apidae). Suom. Hymnteistietellinen Aikakausk. 44, 10-26.

Mooney, H.A., Cleland, E.E., 2001. The evolutionary impact of invasive species. Proc. Natl. Acad. Sci. U. S. A. $98,5446-51$.

Murray, T.E., Coffey, M.F., Kehoe, E., Horgan, F.G., 2013. Pathogen prevalence in commercially reared bumble bees and evidence of spillover in conspecific populations. Biol. Conserv. 159, 269-276.

Oksanen, F.J., Blanchet, G., Kindt, R., Legendre, P., Minchin, P.R., O’Hara, R.B., Simpson, G.L., Solymos, P., Stevens, M.H.H., Wagner, H., 2011. Vegan: Community Ecology Package. Tertiary Vegan: Community Ecology Package.

Paradis, E., Claude, J., Strimmer, K., 2004. APE: Analyses of phylogenetics and evolution in R language. Bioinformatics 20, 289-290.

Peat, J., Darvill, B., Ellis, J., Goulson, D., 2005. Effects of climate on intra- and interspecific size variation in bumble-bees. Funct. Ecol. 19, 145-151.

Perrings, C., Burgiel, S., Lonsdale, M., Mooney, H., Williamson, M., 2010. International cooperation in the solution to trade-related invasive species risks. Ann. N. Y. Acad. Sci. 1195, 198-212.

R Development Core Team, 2013. R: A language and environment for statistical computing, Tertiary R: A language and environment for statistical computing. R Foundation for Statistical Computing, Vienna, Austria.

Rambaut, A., Drummond, A.J., 2007. Tracer. Tertiary Tracer.

Rasmont, P., Coppée, A., Michez, D., De Meulemeester, T., 2008. An overview of the Bombus terrestris (L. 
Reid, N.M., Carstens, B.C., 2012. Phylogenetic estimation error can decrease the accuracy of species delimitation: a Bayesian implementation of the general mixed Yule-coalescent model. BMC Evol. Biol. $12,196$.

Rhymer, J.M., Simberloff, D., 1996. Extinction by hybridization and introgression. Annu. Rev. Ecol. Syst. 27, 83-109.

Schlick-Steiner, B.C., Steiner, F.M., Seifert, B., Stauffer, C., Christian, E., Crozier, R.H., 2010. Integrative taxonomy: a multisource approach to exploring biodiversity. Annu. Rev. Entomol. 55, 421-38.

Schmid-Hempel, R., Eckhardt, M., Goulson, D., Heinzmann, D., Lange, C., Plischuk, S., Escudero, L.R., Salathé, R., Scriven, J.J., Schmid-Hempel, P., 2014. The invasion of southern South America by imported bumblebees and associated parasites. J. Anim. Ecol. 83, 823-37.

Sexton, J.P., McKay, J.K., Sala, A., 2002. Plasticity and genetic diversity may allow saltcedar to invade cold climates in North America. Ecol. Appl. 12, 1652-1660.

Sgrò, C.M., Lowe, A.J., Hoffmann, A.A., 2011. Building evolutionary resilience for conserving biodiversity under climate change. Evol. Appl. 4, 326-337.

Simberloff, D., Nuñez, M.A., Ledgard, N.J., Pauchard, A., Richardson, D.M., Sarasola, M., Van Wilgen, B.W., Zalba, S.M., Zenni, R.D., Bustamante, R., Peña, E., Ziller, S.R., 2009. Spread and impact of introduced conifers in South America: Lessons from other southern hemisphere regions. Austral Ecol. 35, 489-504.

Song, J., Carter, C.A., 1996. Rice trade liberalization and implications for US policy. Am. J. Agric. Econ. 78, 891-905.

Velthuis, H.H.W., van Doorn, A., 2006. A century of advances in bumblebee domestication and the economic and environmental aspects of its commercialization for pollination. Apidologie 37, 421-451.

Vereecken, N.J., Mant, J., Schiestl, F.P., 2007. Population differentiation in female sex pheromone and male preferences in a solitary bee. Behav. Ecol. Sociobiol. 61, 811-821.

Widmer, A., Schmid-Hempel, P., Estoup, A., Scholl, A., 1998. Population genetic structure and colonization history of Bombus terrestris s. 1. (Hymenoptera: Apidae) from the Canary Islands and Madeira. Heredity (Edinb). 81, 563-572. 
505 Williams, P.H., An, J., Brown, M.J.F., Carolan, J.C., Goulson, D., Huang, J., Ito, M., 2012a. Cryptic bumblebee species: consequences for conservation and the trade in greenhouse pollinators. PLoS One 7, e32992.

Williams, P.H., Brown, M.J.F., Carolan, J.C., An, J., Goulson, D., Aytekin, A.M., Best, L.R., Byvaltsev, A.M., Cederberg, B., Dawson, R., Huang, J., Ito, M., Monfared, A., Raina, R.H., Schmid-Hempel, P., Sheffield, C.S., Šima, P., Xie, Z., 2012b. Unveiling cryptic species of the bumblebee subgenus Bombus s. str. worldwide with COI barcodes (Hymenoptera: Apidae). Syst. Biodivers. 10, 21-56.

511 Žáček, P., Kalinová, B., Šobotník, J., Hovorka, O., Ptácek, V., Coppée, A., Verheggen, F., Valterová, I., 2009. terrestris and Bombus lucorum. J. Chem. Ecol. 35, 698-705.

514 
516 Table 1 Current taxonomic status according to Rasmont et al. 2008 and Williams et al. 2012b, and proposed 517 taxonomic status. Color pattern is indicated only when a taxon has an original color pattern (+/-means that the 518 taxon has/ has not a specific color pattern; ++ means a very conspicuous original color pattern). Private 519 haplotypes indicates if a taxon has an original haplotype (+/-means that the taxon has/ has not only private 520 haplotype(s)). bGMYC indicates if a taxon has a low probability to be conspecific with other taxa according to 521 bGMYC analyses (+/-means that the taxon has a probability $<0.05 />0.05$ to be conspecific with other taxa).

522 When the taxon is not regarded as a prospective species, the number in square brackets indicates the number of 523 taxa recognized as conspecific according to bGMYC methods. CLGS indicates if the taxon has/has not specific 524 composition of cephalic labial gland secretions (+/-means that the taxon has/ has not a specific CLGS 525 composition according to multiple response permutation procedure analyses. ++ means that the specific composition involved main compounds. When the taxon shares CLGS composition with other ones, the number in square brackets indicates the number of taxa that share similar CLGS) while the letter in square brackets indicates the letter of taxa that share same main compounds.

529

\section{Current taxonomic status}

B. terrestris africanus

B. terrestris audax

B. terrestris calabricus

B. terrestris canariensis

B. terrestris dalmatinus

B. terrestris lusitanicus

B. terrestris sassaricus

B. terrestris terrestris

B. terrestris xanthopus / B. xanthopus

B. ignitus

\section{Color Private pattern haplotypes}

$\begin{array}{ccccl}+ & + & -[1] & +[\mathrm{A}] & \text { B. terrestris africanus } \\ + & -[1] & -[1] & -[1][\mathrm{A}] & \text { B. terrestris audax } \\ + & -[1] & -[1] & -[1][\mathrm{A}] & \text { B. terrestris calabricus } \\ ++ & + & -[1] & +[\mathrm{A}] & \text { B. terrestris canariensis } \\ + & -[1] & -[1] & -[1][\mathrm{A} / \mathrm{B}] & \text { B. terrestris dalmatinus } \\ ++ & -[1] & -[1] & -[1][\mathrm{A}] & \text { B. terrestris lusitanicus } \\ ++ & + & -[1] & +[\mathrm{B}] & \text { B. terrestris sassaricus } \\ + & -[1] & -[1] & -[1][\mathrm{A}] & \text { B. terrestris terrestris } \\ ++ & + & + & ++ & \text { B. xanthopus } \\ ++ & + & + & ++ & \text { B. ignitus }\end{array}$


533 Fig. 1 Bombus terrestris taxa, their geographic distribution (around 1985), and the closely related species

534 Bombus ignitus. A. Bombus terrestris xanthopus. B. Bombus terrestris africanus. C. Bombus terrestris audax. D.

535 Bombus terrestris canariensis. E. Bombus terrestris dalmatinus. F. Bombus terrestris lusitanicus. G. Bombus

536 terrestris sassaricus. H. Bombus terrestris terrestris. I. Bombus ignitus. J. Distribution of Bombus terrestris taxa

537 (modified from Rasmont et al., 2008). All photos by P. Rasmont to the exception of A. and H. by T. Lecocq.

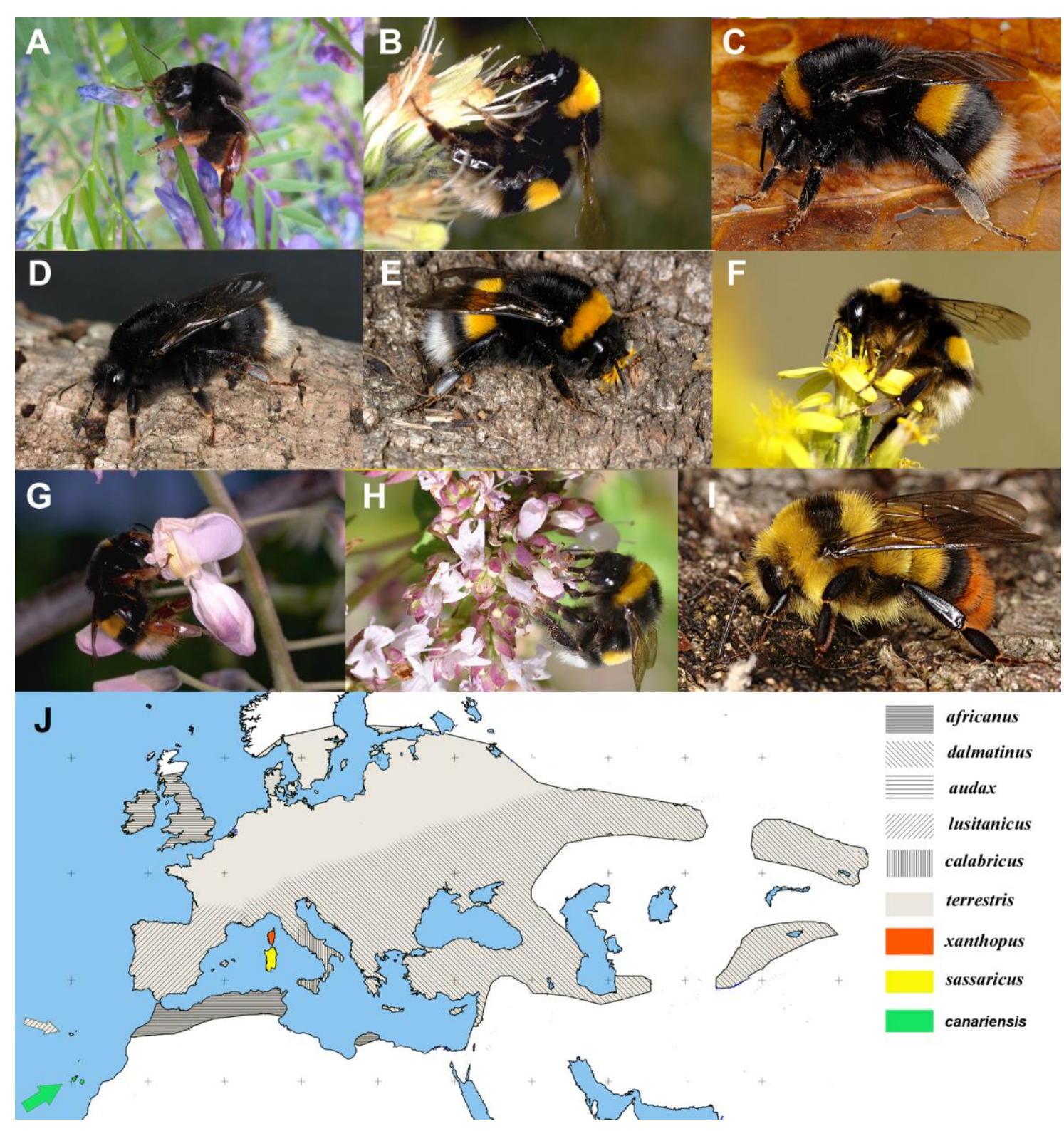


540 Fig. 2 Phylogenetic, DNA sequence-based species delimitation, and cephalic labial gland secretion analysis of 541 Bombus terrestris taxa and Bombus ignitus. A. Bayesian ultrametric tree of Bombus terrestris taxa and B. ignitus 542 based on COI sequences with bGMYC (Bayesian implementation of the general mixed Yule-coalescent) 543 pairwise probability of conspecificity. Values above tree branches are Bayesian posterior probabilities values. 544 Only posterior probabilities $>0.95$ are showed. The colored matrix corresponds to the pairwise probabilities of 545 conspecificity returned by the bGMYC method (see also the related color scale on the right). B. Unweighted pair 546 group method with arithmetic mean (UPGMA) cluster based on a correlation matrix calculated from the cephalic 547 labial gland secretions matrix of Bombus terrestris taxa and B. ignitus. The values near nodes are multiscale 548 bootstrap resampling (only values $>90$ of main groups are shown). 

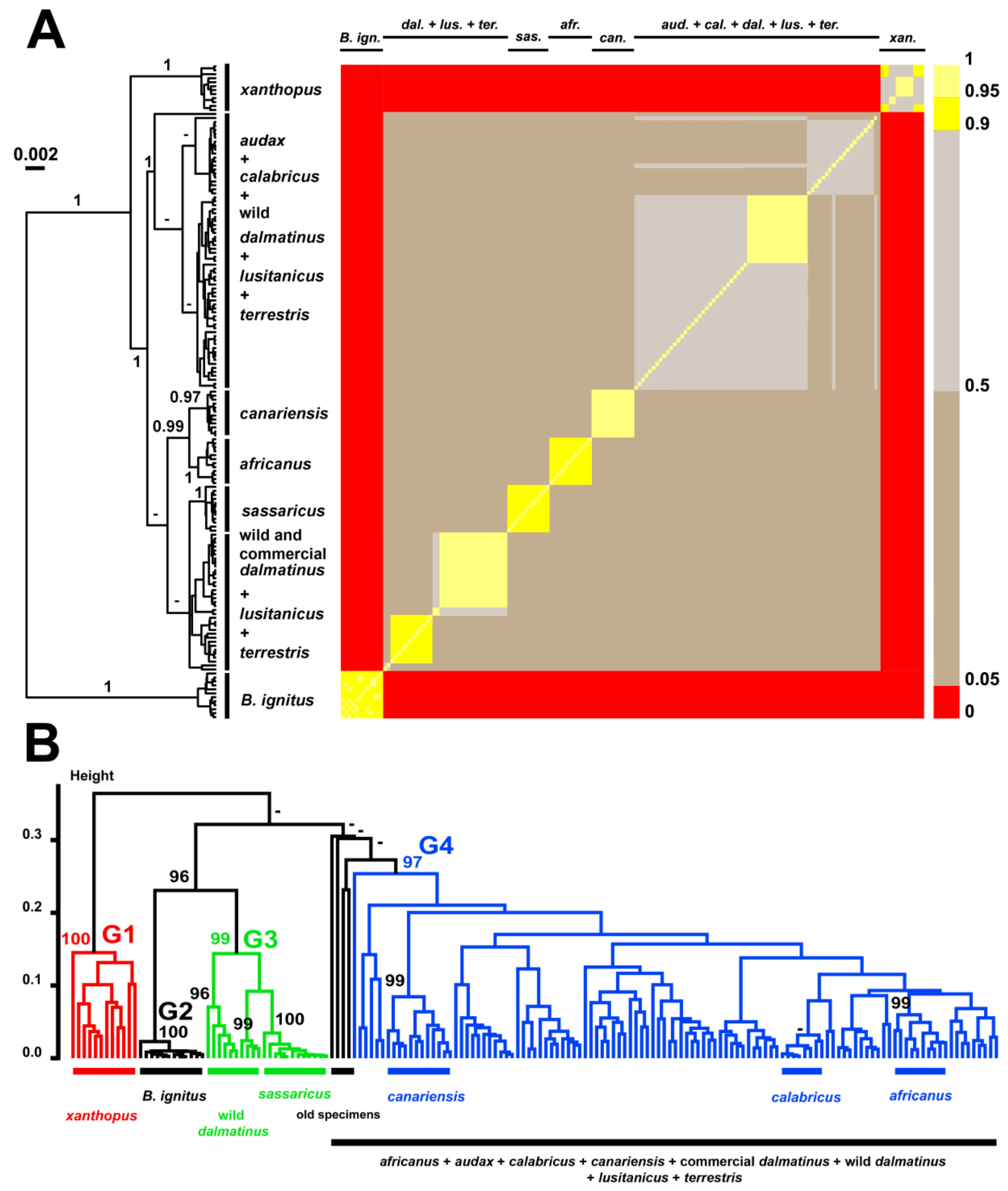
552 Appendix A Table A1 Table of sampling of Bombus terrestris and Bombus ignitus. Sample Code is the sample 553 labels used in analyses. Taxa column is the name of taxa. COI is the Genbank accession numbers for each 554 sample (when consubspecific samples display the same gene sequence, only one of them has been submitted to 555 Genbank).

556 Appendix A Table A2 Cephalic labial gland matrix. Data matrix of cephalic labial gland secretions (relative 557 amounts of each compound) and list of the identified compounds in Bombus terrestris and Bombus ignitus.

558 Unknown $\mathrm{x}$ are undetermined compounds. IndVal results are indicator value obtained in IndVal method.

559 Appendix A Table A3. Sequence divergences between Bombus terrestris taxa.

560 Appendix A Table A4 Results of the bGMYC (Bayesian implementation of the general mixed Yule-coalescent) 561 analysis (pairwise table) in Bombus terrestris and Bombus ignitus. Values are probability to be conspecific $(1=$ $562100 \%$ of chance to be conspecific). 